\title{
Identification of pregnancy-associated plasma protein A as a migration-promoting gene in malignant pleural mesothelioma cells: a potential therapeutic target.
}

\author{
Jun Huang ${ }^{1}$, Sho Tabata ${ }^{1}$, Soji Kakiuchi ${ }^{1}$, Trung The Van ${ }^{1}$, Hisatsugu Goto ${ }^{1}$, Masaki \\ Hanibuchi ${ }^{1}$, Yasuhiko Nishioka ${ }^{1}$ \\ ${ }^{1}$ Department of Respiratory Medicine and Rheumatology, Institute of Health Biosciences, The University of Tokushima \\ Graduate School, Tokushima, Japan \\ Correspondence to: Yasuhiko Nishioka, email: yasuhiko@clin.med.tokushima-u.ac.jp \\ Keywords: malignant pleural mesothelioma, pregnancy-associated plasma protein A, migration, orthotopic xenograft mouse \\ model \\ Received: June 30, 2013 \\ Accepted: July 6, 2013 \\ Published: July 8, 2013
}

This is an open-access article distributed under the terms of the Creative Commons Attribution License, which permits unrestricted use, distribution, and reproduction in any medium, provided the original author and source are credited.

\section{ABSTRACT:}

Despite recent advances in treatment, malignant pleural mesothelioma (MPM) remains a deadly disease. Targeted therapy generated broad interests and is highly expected for the treatment of MPM, yet promising preclinical results have not been translated into substantial clinical benefits for the patients. In this study, we tried to identify the genes which play functional roles in cell migration as well as to test whether they can be used as novel targets for molecular targeted therapy for MPM in preclinical model. In our study, pregnancy-associated plasma protein A (PAPPA) was identified as a gene whose expression level is correlated with MPM cell migration by correlation analysis combining MPM cell migration ability and their gene expression profiles. Highly migratory cells were selected from MPM cell lines, MSTO-211H, NCI-H290 and EHMES-1 in vitro and up-regulation of PAPPA in these cells were confirmed. In vitro, PAPPA was demonstrated to stimulate the MPM cell migration via cleavage of insulin-like growth factor-binding protein-4 and subsequent release of IGF-1. Gene silencing of PAPPA in MPM cells led to reduced migration, invasion and proliferation. Furthermore, PAPPA shRNA transfected NCI-H290 when orthotopically inoculated into pleural cavity of severe combined immunodeficiency recipient mice, failed to develop tumors and produce bloody pleural effusion as control shRNA transfected cells did. Our study suggests that PAPPA plays a functional role in promoting MPM cell migration and it might serve as a potential therapeutic target for the treatment of MPM.

\section{INTRODUCTION}

\footnotetext{
Malignant pleural mesothelioma (MPM), the most common mesothelioma, is an aggressive tumor associated with prior asbestos exposures that arise from mesothelial surface of pleura [1]. Despite recent advances in treatment, MPM remains a deadly disease. The benefit from surgery is confined to a minority of patients. In addition, cytotoxic chemotherapeutic drugs improve quality of life for unresectable patients, and can prolong survival, though only by a few months at best [2]. There has been substantial interest in the role of targeted agents in the treatment of MPM, a variety of them has been tested as
}

therapy for this malignancy in preclinical studies [3-5], yet promising preclinical data has not been translated into clinical benefits in a significant way [6]. More about the biology of this disease might be needed for the molecular targeted therapy to yield promising new treatment options.

Even though metastases are rarely the cause of death of MPM patients, the unique clinical feature of local spread along tissue planes not only causes increasing pain and functional abnormalities, but also makes it a difficult neoplasm to manage $[1,7]$. Cell migration as a key to local invasion, might provide an opening for therapeutic approaches if we can understand more about the molecular biology that underlie it [8]. The goal of our study was 
to identify the genes which might be involved in the regulation of cell migration as well as to evaluate whether they can be used as targets for the inhibition of MPM cell migration, invasion or even tumor progression.

To achieve this goal, we investigated the migration ability of 8 human MPM cell lines in vitro and their gene expression profiles. Genes whose expressions correlated with cell migration were identified by correlation analysis combining the migration ability of MPM cells and their gene expression profiles. Among these genes, pregnancyassociated plasma protein A (PAPPA) caught our attention. $P A P P A$, one of four proteins of placental origin found circulating at high concentrations in pregnant women [9], is now basically recognized as a local regulator of Insulinlike growth factor 1 (IGF-1) bioavailability through cleavage of IGF-binding proteins (IGFBPs) [10-12]. The IGF axis was reported to be involved in the pathogenesis of many cancer types [13], including MPM [14-16], and therapeutics aimed at the IGF axis are under extensive clinical investigation [17]. Accumulating preclinical evidences support the idea that PAPPA may play a role in cancer [18-22].

In the present study, a series of in vitro assay was performed to try to reveal the mechanism of the migrationpromoting role of $P A P P A$. To further confirm the causal role of $P A P P A$ in MPM cell migration, we applied both small interfering RNA (siRNA) and small hairpin RNA (shRNA) targeting $P A P P A$ to investigate the silencing effects of $P A P P A$ on MPM cell migration. Finally, we used an orthotopic xenograft mouse model of MPM [3-5, $24]$ to evaluate the efficacy of $P A P P A$ silencing on tumor development and progression in vivo.

\section{RESULTS}

\section{Expression of PAPPA is correlated with migration ability in MPM cell lines}

To identify potential candidate genes whose function are related with MPM cell migration, we determined the migration ability of eight MPM cell lines using transwell chamber migration assay (Fig. 1A), and obtained their gene expression profiles using Affymetrix whole-genome oligonucleotide microarray (Supplementary Fig. 1A). After we applied Pearson's correlation by combining the migration ability of MPM cell lines and their gene expression profiles, expression levels of several genes revealed relatively high correlations with MPM cell migration ability (Supplementary Fig. 1B). Among these genes, we focused on $P A P P A$, which encodes a secreted metalloproteinase that degrades inhibitory IGFBPs. We validated our microarray expression data for $P A P P A$ with quantitative real time PCR (qRT-PCR) (Fig. 1B). Overall, $P A P P A$ expression results generated from the two different techniques were well correlated (Supplementary Fig. 1B), except that of NCI-H28. Thus we did not include NCI-H28 in the following studies. The similarity of expression levels of PAPPA obtained from microarray and qRT-PCR can also be extended to their correlations with MPM cell migration ability (Supplementary Fig. 1B, Fig. 1C). Considering that PAPPA functions as a secreted protein, we further determined the secreted protein levels of PAPPA in the conditioned medium (48 h) of MPM cell culture (Fig. 1D). The secreted PAPPA levels of MPM cell lines are also tend to be positively correlated with their migration ability (Fig. 1E, Pearson $\mathrm{r}=0.7217, P=$ $0.0671)$.

Considering the possibility that the correlation is simply due to coincidence, rather than the functional role of PAPPA in the regulation of MPM cell migration, we applied an in vitro selection strategy to further examine this correlation (Fig. 2A). Highly migratory MPM cells were selected, and their gene expression levels were examined. Three MPM cell lines, MSTO-211H, NCI-H290 and Y-MESO-14, after 3-5 round in vitro selection, increased their migration ability roughly 5 -fold compared to their corresponding parental cell lines (Fig. 2B). Interestingly, when analyzing their gene expression profiles with microarray, we found that the genes including $P A P P A$, which we previously identified whose expression levels were positively correlated with migration ability (Supplementary Fig. 1B), were also expressed moderately higher in selected highly migratory cells as compared with their parental cell lines (Supplementary Table 1, Supplementary Fig. 3). Increased expression of PAPPA in highly migratory cells was further validated by qRT-PCR (Fig. 2C). These observations strongly indicated that there is an inherent relationship between PAPPA expression and migration ability in MPM cells.

\section{Major components of the IGF system are expressed in MPM cell lines}

PAPPA has long been identified as a local regulator of IGF bioavailability through cleavage of IGFBP4 [1012], while IGF-1 has already been reported that it can induce MPM cell migration, a biological effect associated with IRS-2 phosphorylation [15]. We speculated that PAPPA may play a functional role in regulation of migration of MPM cells, at least partially, through the IGFBP-4/IGF-1/IGF-1R1 axis. Thus we characterized the major components of the IGF axis in MPM cell lines we used. IGF-1R expressions were detected at both mRNA and protein levels (Fig. 3A, 3B), which is consistent with previous report [23]. For IGF-1, no significant levels of secreted IGF-1 were detected by ELISA, and actually no mRNA of $I G F 1$ was detected by qRT-PCR (data not shown). For IGFBPs, we examined the mRNA transcript profiles of $I G F B P 1$ to 5 of MPM cells, notably, 
IGFBP3 and IGFBP4 were found to be the dominant IGF-1 binding proteins (Fig. 3C) expressed in MPM cell lines. We subsequently measured the concentrations of secreted IGFBP-3 (Fig. 3D) and IGFBP-4 (Fig. 3E) in the conditioned medium of MPM cell cultures. To test whether the PAPPA detected in the MPM cell conditioned medium is enzymatically active, we incubated rhIGFBP-4, rhIGF-1 with conditioned medium for $24 \mathrm{~h}$, and subjected the conditioned medium to immunoblotting analysis. The conditioned medium of MPM cells did reveal enzymatic activity, as indicated by reduced rhIGFBP-4 after incubation (Fig. 3F). And the enzymatic activity of the conditioned medium seems to be correlated with the concentrations of PAPPA in these medium (Fig. 3F, Fig. 1D). Since tissue plasminogen activator (tPA) was reported to have IGFBP-3 proteolytic activity [24], and we also detected that PLAT (gene encoding tPA protein) is expressed in MPM cell lines (Supplementary Fig. 1B), we performed an in vitro proteolysis analysis to see whether the enzymatic cleavage of IGFBP-4 of conditioned medium of MPM cells can also be attributed to tPA. It turned out that PAPPA revealed proteolytic activity of IGFBP-4 but not IGFBP-3, while tPA revealed proteolytic activity of IGFBP-3 but not IGFBP-4 (Fig. 3G), supporting the idea that PAPPA is specifically responsible for the IGFBP-4 proleolytic activity of MPM cell conditioned medium. Taken together, the major components of the IGF axis are expressed in MPM cells, which gives the context of functional role of PAPPA as local regulator of IGF-1 for MPM cells.
A.

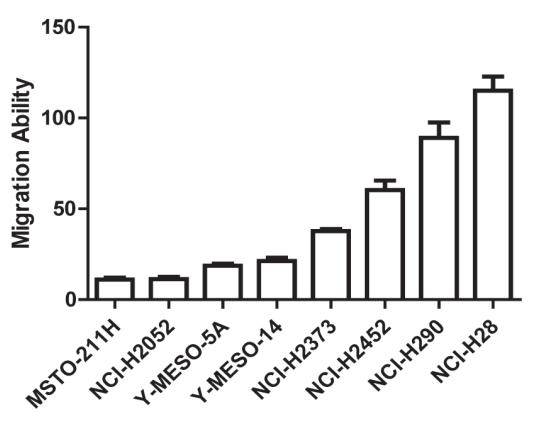

B.

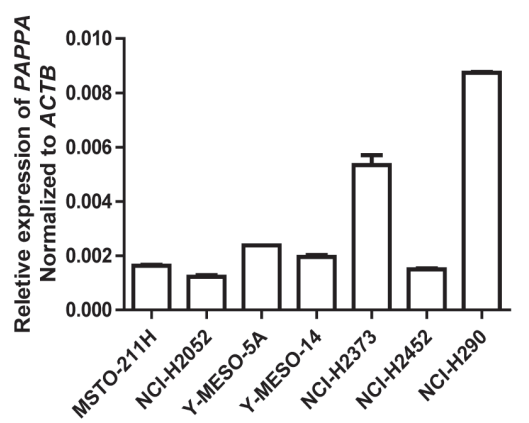

D.

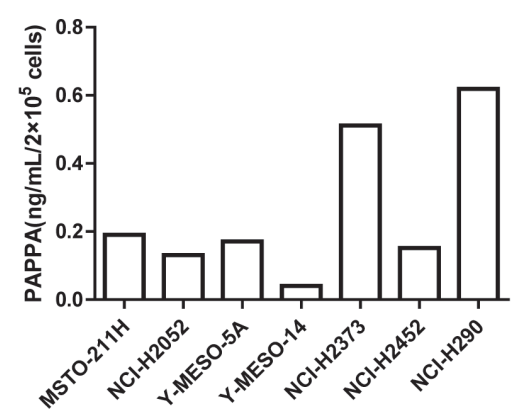

c.

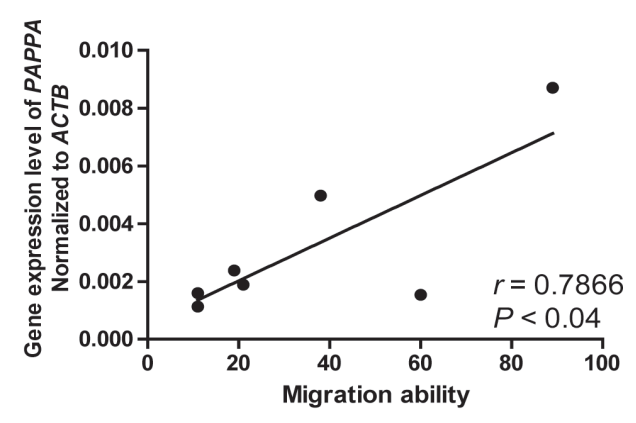

E.

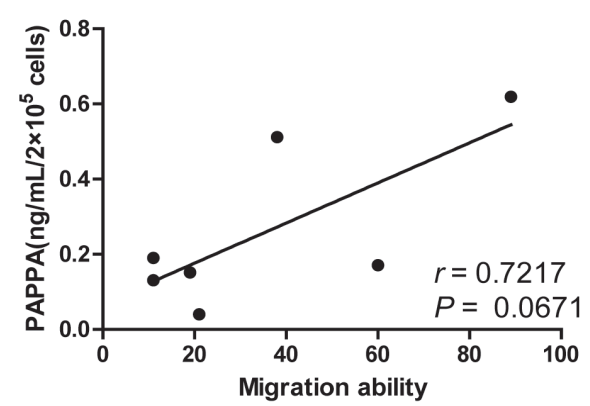

Figure 1: Migration abilities of malignant pleural mesothelioma cells are positively correlated with their expression levels of PAPPA. A, The migration abilities of eight MPM cell lines determined by transwell migration assay, data are the means \pm SEMs of three independent experiments. B, The expression levels of PAPPA in eight MPM cell lines determined by qRT-PCR. Data are representative of two independent assays carried out in triplicate (means $\pm \mathrm{SDs}, \mathrm{n}=3$ ). $\mathrm{C}$, The expression levels of $P A P P A$ in eight MPM cell lines determined by qRT-PCR, were positively correlated with their migration ability. $* P<0.05$. The correlation efficient (r) and the significance $(P)$ were calculated by Pearson's correlation analysis. D, The concentrations of secreted PAPPA in the conditioned medium $(48 \mathrm{~h})$ were determined by ELISA. E, The concentrations of secreted PAPPA from MPM cells (D) tend to have positive correlation with their migration abilities. $P=0.0671$. The correlation efficient $(r)$ and the significance $(P)$ were calculated by Pearson's correlation analysis. 
PAPPA enhanced migration of MPM cells by enzymatically cleaving inhibitive IGFBP-4 and releasing IGF-1 as chemotactic factor

To test the hypothesis that PAPPA could affect MPM cell migration via IGFBP-4/IGF-1/IGF-1R axis, a series of experiments were performed using transwell chamber assays with related recombinant proteins. Consistent with previous reports [15, 25], IGF-1 alone could stimulate the migration of EHMES-1 and MSTO-211H cells across a porous membrane in a dose-dependent manner (Fig. 4A, Fig. 4C). We also demonstrated that stimulating effect of IGF-1 could be blocked by IGFBP-4, while further addition of PAPPA would recover the stimulating effects of IGF-1 on MPM cell migration (Fig. 4B, Fig. 4D). PAPPA alone added to the lower chamber has no effects on cell migration (data not shown), excluding the possibility that the enhanced migration of MPM was due to the direct chemotactic effect of PAPPA. To further confirm that the released IGF-1, rather than the fragments of IGFBP-4 generated by cleavage, recovered the enhanced cell migration, anti-IGF-1 antibody was used to neutralize the released IGF-1 from IGFBP-4 proteolysis caused by
PAPPA. The enhanced cell migration was completely abrogated by anti-IGF-1 antibody (Fig. 4E). Taken together, our results suggest that PAPPA could enhance the migration of MPM cell via proteolysis of IGFBP-4 and subsequently releasing IGF-1 as chemotactic factor.

\section{Silencing PAPPA by small interfering RNA inhibited migration and proliferation of MPM cells}

To investigate the function of endogenous PAPPA on MPM cell migration more directly, we knocked down PAPPA in MPM cells (NCI-H290, MSTO-211H and EHMES-1) using siRNA and subsequently evaluated their migration ability. Efficacy of PAPPA silencing by siRNA were confirmed by qRT-PCR before the following functional assays (Fig. 5A). We found that PAPPA silencing significantly reduced cell migration (Fig. 5B). No difference of cell viability was observed at the time point of migration (24-30 h after siRNA treatment), as revealed by MTT assay (data not shown), but the inhibition of cell growth was also observed later, which became evident $72 \mathrm{~h}$ after PAPPA siRNA treatment (Fig.

A.

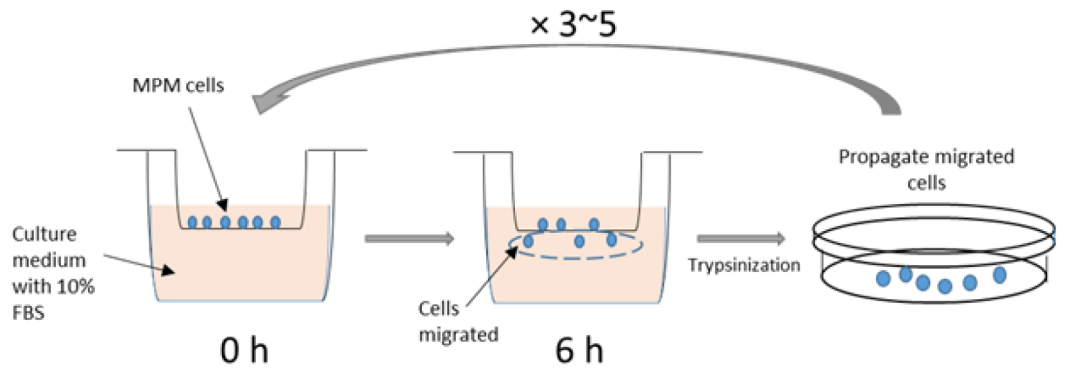

B.
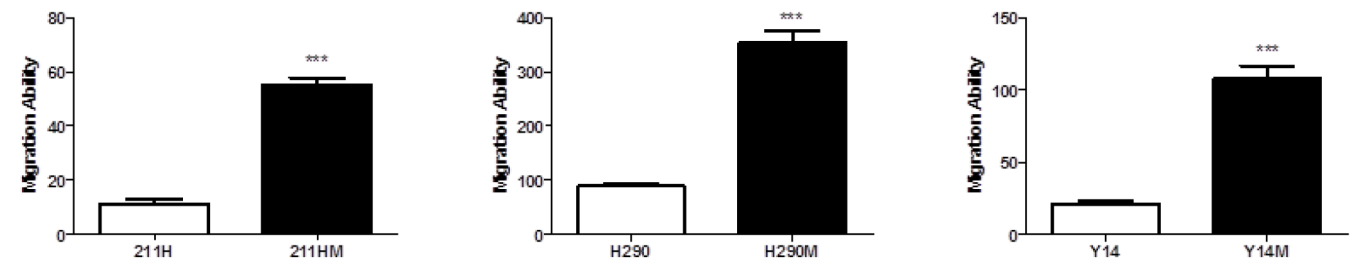

C.
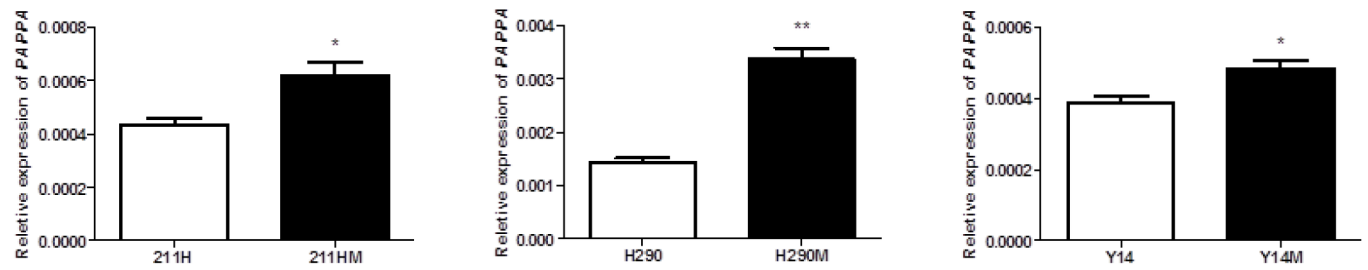

Figure 2: Selected highly migratory MPM cells and their PAPPA expression levels. A, Schematic representation of in vitro selection of highly migratory cells. B, The cells that went through in vitro selection process revealed higher migration ability (means \pm $\mathrm{SDs}, \mathrm{n}=3)$. Left, NCI-211H parental cell $(211 \mathrm{H})$ versus NCI-211 highly migratory cells $(211 \mathrm{HM})$. *** $P<0.001$, unpaired $t$ test; middle, NCI-H290 parental cell (H290) versus NCI-H290 highly migratory cells (H290M). ${ }^{* * *} P<0.001$, unpaired $t$ test; right, Y-MESO-14 parental cells (Y14) versus Y-MESO-14 highly migratory cells (Y14M). *** $P<0.001$, unpaired $t$ test. C, The expression levels of PAPPA in selected cells were significantly higher as compared with that in parental cells. The data are representative of two independent assays carried out in triplicate (means $\pm \mathrm{SDs}, \mathrm{n}=3$ ). Left, $211 \mathrm{H}$ versus $211 \mathrm{HM}$. $* P<0.05$, unpaired $t$ test; middle, $\mathrm{H} 290$ versus $\mathrm{H} 290 \mathrm{M}, * P<0.05$, unpaired $t$ test; right, Y14 versus Y14M, * $P<0.05$, unpaired $t$ test. 
5C). These data confirmed the functional roles of $P A P P A$ on the migration of MPM cells and revealed an extended role of PAPPA on the proliferation of these cells, indicating it might be directly used as therapeutic target to inhibit progression of MPM in vivo.
A.

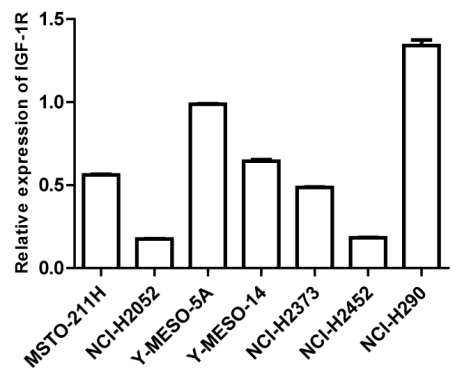

C.

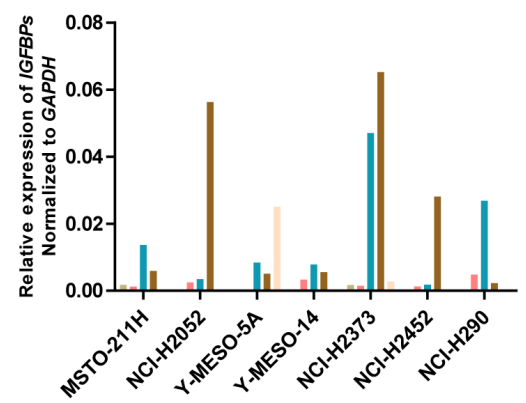

E.

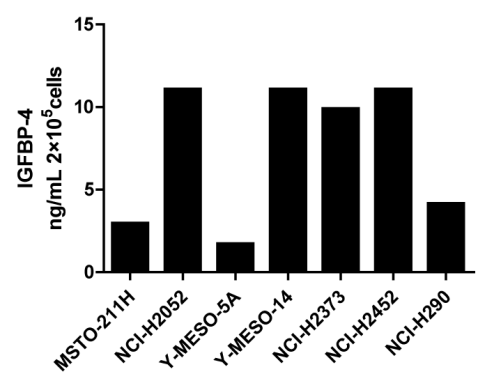

G.

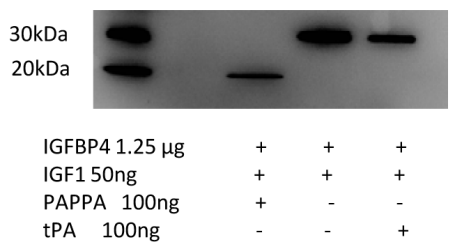

B.

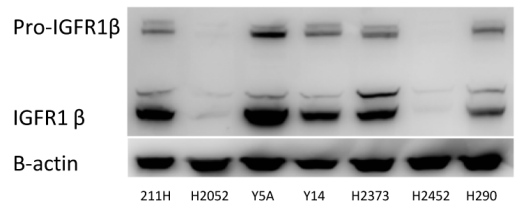

D.

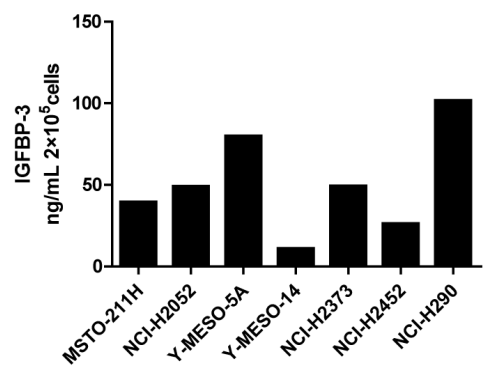

F.

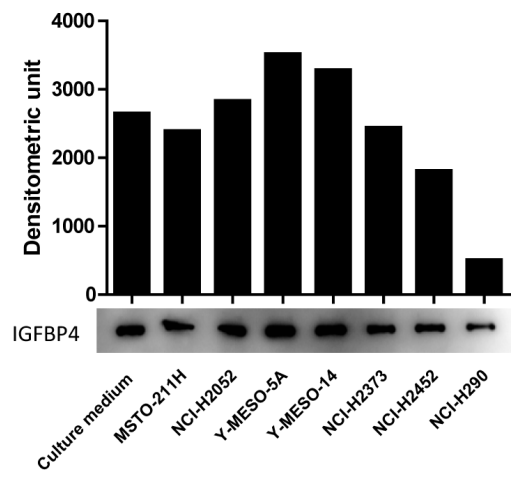

$30 \mathrm{kDa}$ $20 \mathrm{kDa}$

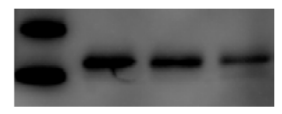

IGFBP3

Figure 3: Characterization of major IGF components expression in MPM cell lines. A, Relative gene expression levels of IGF-1R in MPM cell lines assessed by qRT-PCR, normalized to the relative expression of IGF-1R in Y-MESO-5A cells. The data are representative of two independent assays carried out in triplicate (means $\pm \mathrm{SDs}, \mathrm{n}=3$ ). B, IGF-1R1 expression detected by immunoblotting. $\mathrm{C}$, Relative gene expression levels of IGFBPS in MPM cell lines characterized by qRT-PCR, normalized to that of $A C T B$. D, The concentrations of secreted IGFBP-3 in the conditioned medium $(24 \mathrm{~h})$ of MPM cells detected by ELISA. E, The concentrations of secreted IGFBP-4 in the conditioned medium ( $24 \mathrm{~h}$ ) of MPM cells by ELISA. F, The conditioned medium of MPM cell lines revealed enzymatic activity of cleaving IGFBP-4. For the detection of IGFBP-4 proteolysis, rhIGFBP-4 (0.5 $\mu \mathrm{g})$, rhIGF-1 (100 ng) were add into $50 \mu 1$ conditioned medium of MPM cells and incubated for overnight at $37^{\circ} \mathrm{C}$, then conditioned medium was subjected to immunoblotting analysis. G, The enzymatic activity of conditioned medium of mesothelioma cell lines. Left panel, rhIGF-1, rhIGFPB-4 was incubated with or without rhPAPPA for $24 \mathrm{~h}$, and then subjected to immunoblotting analysis. rhPAPPA but not rhtPA revealed specific enzymatic activity of cleavage of IGFBP4. Right panel, rhtPA specifically cleaved rhIGFBP-3. 
Transfection of PAPPA shRNA inhibited migration, invasion and proliferation of NCI-H290 cell line in vitro and tumor growth in orthotopic xenograft model

Our next series of experiments were aimed at further determining the phenotypic effects on MPM cells from $P A P P A$ knockdown by establishing stable PAPPA

A.

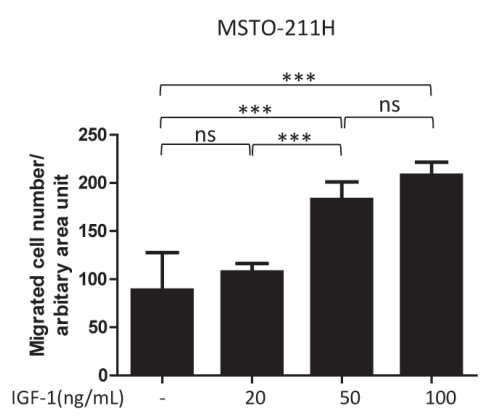

C.

EHMES-1

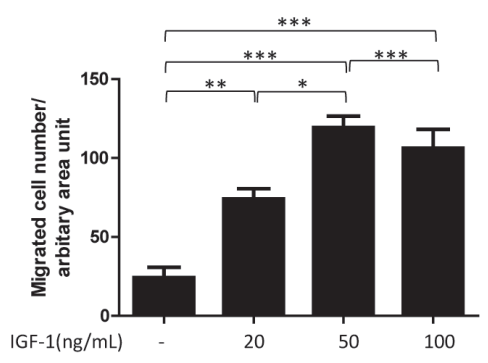

E.

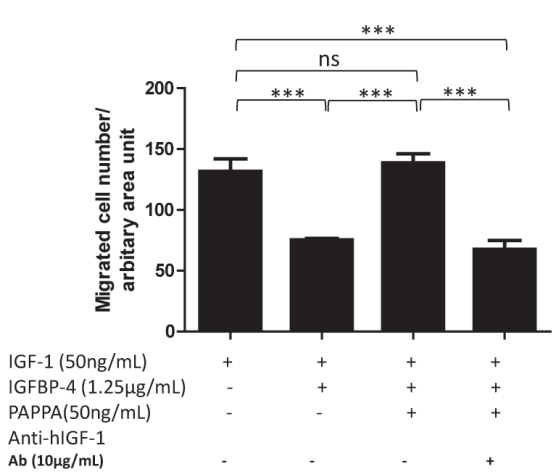

knockdown cell lines. To establish stable cell line, we chose MPM cell line NCI-H290, which can be used to establish an orthotopic xenograft model of human MPM. This model reflects the clinical features of MPM patients, such as local tumor progression, malignant bloody pleural effusion in the pleural cavity [3-5, 26]. Silencing of PAPPA expression in cells transfected with PAPPA shRNA was confirmed (Fig. 6A) before carrying out following assays. Consistent with the results of transient knockdown of

B.

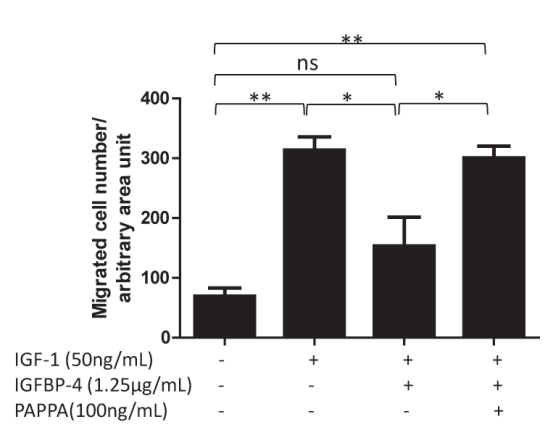

D.

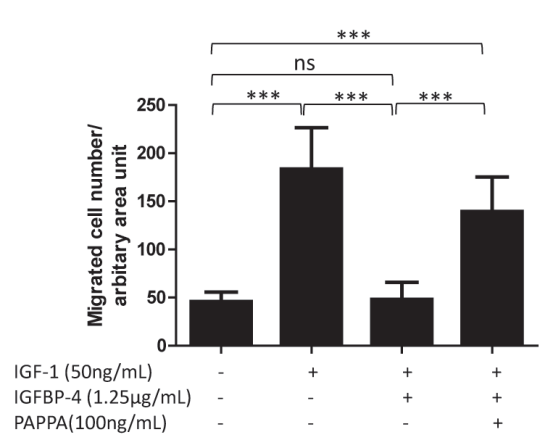

Figure 4: PAPPA enhances migration of MPM cells by enzymatically cleaving inhibitive IGFBP-4 and releasing IGF1 as chemotactic factor. A, rhIGF-1 induced migration of MSTO-211H cells in a dose-dependent manner. B, rhIGFBP-4 inhibited migration of MSTO-211H cells induced by rhIGF-1, while rhPAPPA recovered the ability of rhIGF-1 to induce migration of MSTO-211H. rhIGF-1 or rhIGF-1 + rhIGFBP-4 or rhIGF-1 + rhIGFBP4 + rhPAPPA were added in serum free medium and incubated at $37^{\circ} \mathrm{C}$ for $6 \mathrm{~h}$ before added into the lower chamber. C, rhIGF-1 attracted EHMES-1 cells to migrate dose-dependently. D, rhPAPPA recovered the ability of rhIGF-1 to induce the migration of EHMES-1 which was inhibited by rhIGFBP-4. E, An anti-IGF-1 antibody blocked the reversing effect of rhPAPPA on the migration of EHMES-1. Six-hours after the incubation with the rhIGF-1, rhIGFBP-4 and rhPAPPA mixture, an anti-IGF-1 antibody was added into the mixture and incubated for another $30 \mathrm{~min}$ before being added into the lower chambers. The data are representative of at least two independent assays carried out in triplicate (means $\pm \mathrm{SDs}, \mathrm{n}=3$ ). ${ }^{*} P<0.05,{ }^{* *} P<0.01, * * * P<0.001, \mathrm{~ns}=$ not significant, one way ANOVA analysis. 
PAPPA by siRNA, migration ability of $P A P P A$ shRNA cells was substantially reduced as compared to that of control shRNA cells (Fig. 6B). We also assessed the invasiveness of PAPPA shRNA cells using a Matrigel-coated transwell invasion assay. The decrease of invasiveness of $P A P P A$ shRNA cells until $20-30 \%$ was even more profound than that of their migratory motility until $30-40 \%$ as compared to control shRNA cells (Fig. 6B, Fig. 6C). Also consistent with siRNA experiments, we observed a retardation of proliferation of PAPPA shRNA cells in vitro, as revealed by MTT assay (Fig. 6D). Despite of the growth retardation observed, the cells seem to be viable for a long time (for at least 14 days we followed) as assessed by morphological observation and Trypan blue exclusion test (data not shown).

Finally, we used a mouse orthotopic xenograft model to investigate the roles of PAPPA in MPM tumor progression in vivo. NCI-H290 cells stably transduced with $P A P P A$ shRNA or control shRNA were orthotopically implanted into the pleural cavity of SCID mice. Mice were sacrificed 21-23 days after tumor cell inoculation. Control shRNA NCI-H290 developed tumors in the pleural cavity in all recipient mice at the time point of sacrifice, and showed classical patterns of diffusely grown pleuralbased masses. Four out of five mice developed bloody pleural effusions (hemothorax). On the other hand, PAPPA
shRNA NCI-H290 only developed traces of tumor mass, and almost no pleural effusions were observed (Fig. 6E, Fig. 6F). Taken together, transfection of PAPPA shRNA in MPM cells confirmed the important role of PAPPA in cell migration, invasion and proliferation, further revealed its crucial role in MPM tumor development and progression in vivo.

\section{DISCUSSION}

In this study, we identified $P A P P A$ as a migrationpromoting gene in MPM cell lines. By applying a novel approach of combination of transwell migration assay and microarray gene expression profiling, expression level of $P A P P A$ is found to be correlated with migration ability of MPM cell lines. The correlation between $P A P P A$ expression and migration ability in MPM was further supported by an in vitro selection strategy. $P A P P A$ expression was found to be parallelly up-regulated in three migratory cell lines as compared to their corresponding parental cell lines. These results strongly suggested that PAPPA functionally promotes MPM cell migration.

The role of PAPPA as a local regulator of IGF bioavailability through cleavage of IGFBPs has been well documented [10-12], and the IGF axis was identified as one of the molecular networks involved in the formation,
A.

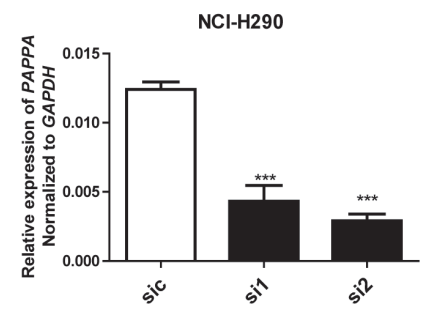

B.

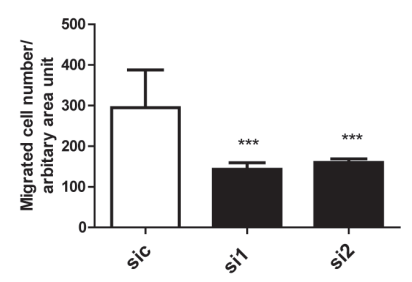

C.

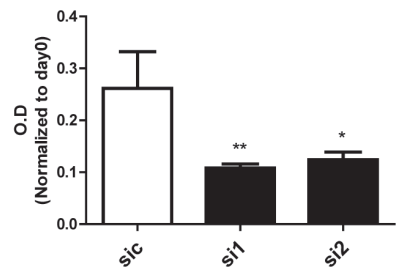

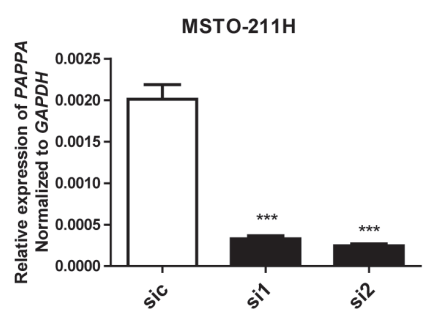
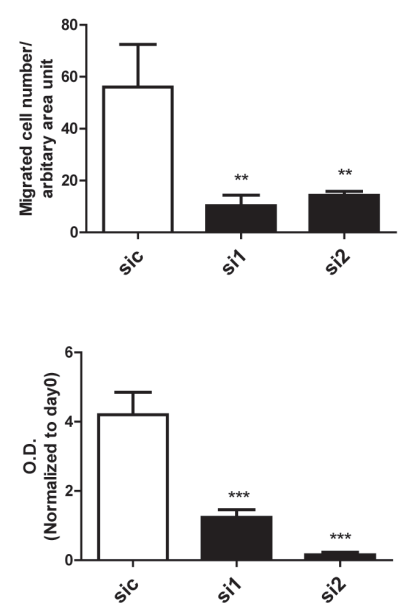

EHMES-1
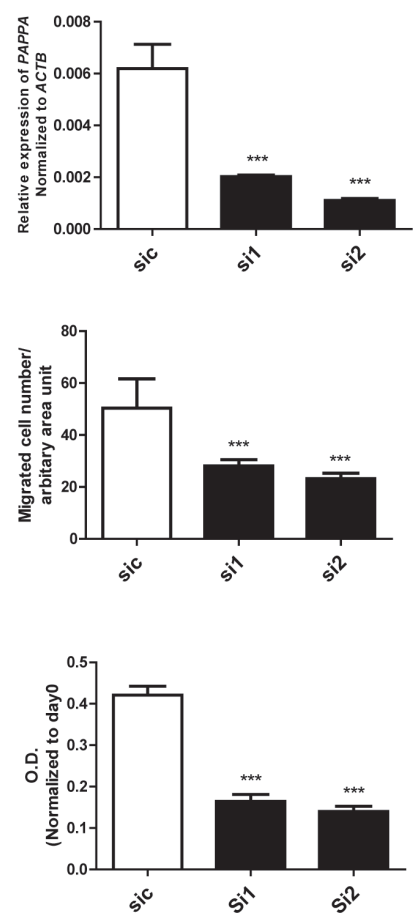

Figure 5: Effects of knockdown of PAPPA by siRNA on migration and proliferation of MPM cells. A, The expression of PAPPA in MPM cells (left, NCI-H290; middle, MSTO-211H; right, EHMES-1) was analyzed by qRT-PCR $24 \mathrm{~h}$ after transfection with siRNA targeting PAPPA. B, The migration ability of MPM cells (left, NCI-H290; middle, MSTO-211H; right, EHMES-1) were determined by transwell migration assay $24 \mathrm{~h}$ after transfection with siRNA targeting PAPPA. C, The proliferation of MPM cells (left, NCI-H290; middle, MSTO-211H; right, EHMES-1) was determined by MTT assay $72 \mathrm{~h}$ after transfection with siRNA targeting $P A P P A$. The data are representative of two independent assays carried out in triplicate (means $\pm \mathrm{SDs}, \mathrm{n}=3$ ), ${ }^{*} P<0.05, * * P<0.01$, *** $P<0.001$, one way ANOVA analysis. 
progression and metastatic spread of many cancer types [13], including MPM [14-16, 27]. Based on this observation, we speculated that PAPPA could promote MPM cell migration via IGF-1. To set the stage for the functional role of $P A P P A$, we characterized the major components of the IGF axis in MPM cell lines. IGF$1 \mathrm{R}$ and IGFBP1-5 were found to be expressed at varied levels in MPM cell lines. Our further experiments clearly demonstrated that the migration-promoting role can be explained by, at least partly, its proteolytic activity of IGFBP-4 to regulate the bioavailability of IGF-1. Previous studies showed the overexpression of IGF-1 in both MPM cell lines and tissues versus nonmalignant mesothelium at transcriptional level $[14,15]$, but we did not detected significant expression of IGF-1 at transcriptional level, which was consistent with another large-scale transcriptional profiling study of MPM [28]. The reason for this discrepancy among different reports remains to be determined, which might be explained by the variety of samples investigated. Despite of the discrepancy, given the fact that physiological ranges of serum IGF1 concentration [29] by far exceed the concentration of IGF-1 needed to have effects on MPM cell migration as demonstrated by us and others $[15,25]$, we believe that

A.

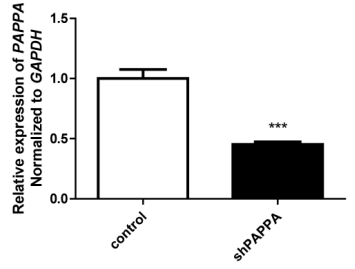

B.

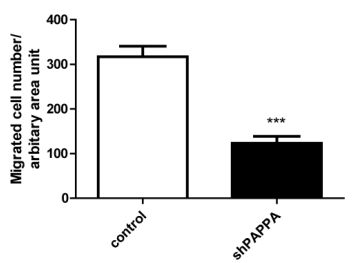

E.

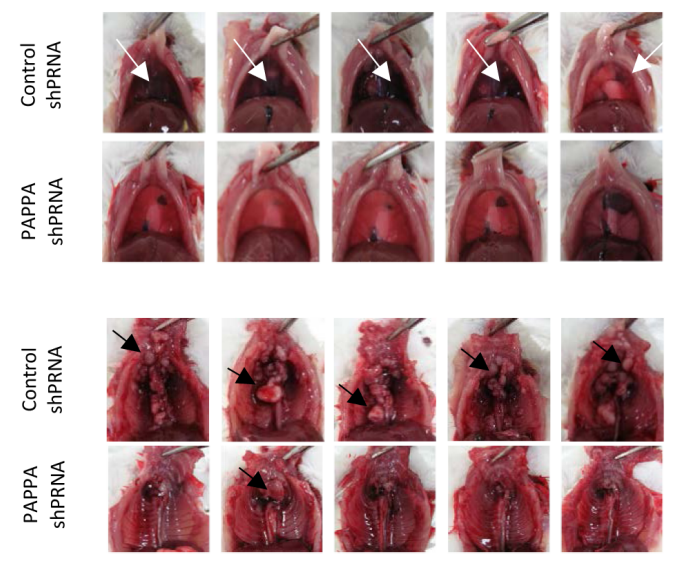

C.

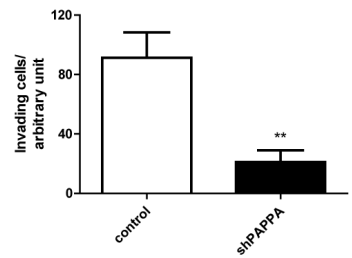

F.
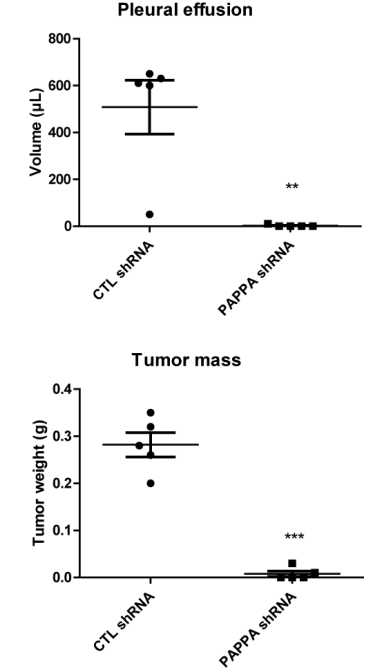

Figure 6: Effects of PAPPA shRNA transfection on migration, invasion, proliferation of NCI-H290 in vitro and tumor growth in orthotopic xenograft model. A, After transfection of $P A P P A$ shRNA, gene silencing efficacy of $P A P P A$ shRNA in NCI-H290 was confirmed by qRT-PCR. $* * * P<0.001$, unpaired $t$ test. B, The migration ability of NCI-H290 cells transfected with $P A P P A$ shRNA was significantly reduced as compared to that of cells transfected with nonsilencing control shRNA. The data are representative of two independent assays carried out in triplicate (means $\pm \mathrm{SDs}, \mathrm{n}=3$ ), $* * * P<0.001$, unpaired $t$ test. $\mathrm{C}$, The cell invasion through the Matrigel was significantly less in PAPPA shPAPPA transfected NCI-H290 cells as compared to that in cell transfected with control shRNA. The data are representative of two independent assays carried out in triplicate (means $\pm \mathrm{SDs}, \mathrm{n}=3$ ), ${ }^{* * *} P<0.001$, unpaired $t$ test. $\mathrm{D}$, The proliferation of $P A P P A$ shRNA transfected cells was inhibited as compared to that of cells transfected with control shRNA. The data are representative of two independent assays carried out in triplicate (means $\pm \mathrm{SDs}, \mathrm{n}=3$ ), $* * * P<0.001$, unpaired $t$ test. E, The effects of $P A P P A$ shRNA on MPM tumor progression in the SCID mouse model of orthotopic xenograft. Pictures of the gross appearance of bloody pleural effusion (upper panel, white arrow) and tumor mass (lower panel, black arrow) of each group were shown. F, The qualitative analysis of pleural effusion (upper panel, $* * P<0.01$, unpaired $t$ test) and tumor mass (lower panel, $* * * P<0.001$, unpaired $t$ test). 
our experiments does have its physiological or even pathogenic relevance. It is worth noting that even though PAPPA did enhance MPM cell migration via IGFBP-4/ IGF-1 axis in our experimental setup, we did not exhaust the possibility that nonproteolytic mechanisms might be involved in the migration-promoting function of $P A P P A$.

In the present study, the functional role of $P A P P A$ in MPM cell migration was investigated by gene silencing experiments using both siRNA and shRNA. Knockdown of PAPPA led to reduced MPM cell migration, invasion and proliferation. Exogenously addition of PAPPA could barely rescue the proliferation of MPM cells from the inhibitory effect of PAPPA silencing in vitro (data not shown). This implies that PAPPA might involve in an additional mechanism which has yet to be elucidated. In addition, NCI-H290 cells transfected with PAPPA shRNA, when inoculated into the pleural cavity of SCID mice, failed to develop tumors in vivo, while NCI-H290 transfected with control shRNA developed massive tumors and bloody pleural effusions. These observations indicate that PAPPA facilitates the progression of MPM and that it might be a potential therapeutic target.

Accumulating evidences support the notion that PAPPA may play a role in cancer. PAPPA knockout mice were observed to have reduced incidence of spontaneous cancers [18]. A tumor growth-promoting role of $P A P P A$ has been demonstrated in breast cancer [20] ovarian cancer [21] and lung cancer [22] with mouse models very recently. Even though the role of PAPPA in cancer has not gathered much attention, IGF-1, the pervasive growth factor whose local bioavailability it regulates, has long been an intense interest for cancer researchers [13]. Actually, several novel therapeutics aimed at the IGF-1/IGF-1R, particularly monoclonal antibodies and small molecule tyrosine kinase inhibitors are under clinical investigation [17]. A phase II clinical trial of cixutumumab, a monoclonal antibody to IGF-IR, which showed encouraging therapeutic efficacy for MPM in preclinical study [23], is currently ongoing for the treatment of patients with mesothelioma. Given the vital physiological function of IGF-1 axis, unforeseen enduring effects on metabolism, body fat, muscle mass, and bone density has been major concerns [17]. In contrast to the pervasive existence and versatile physiological functions of IGF-1 axis, PAPPA is barely expressed in adult tissues except placenta, cardiamyocyte and smooth muscle [30], and actually genetic deletion of PAPPA extended mean and maximum lifespan of by $30-40 \%$ in mice [19]. Therefore, PAPPA might serve as a better therapeutic target for MPM with more tumor specificity and less risks of side effects as compared to IGF-1 axis components as targets. Even though most of the studies on PAPPA so far suggested its tumor-promoting role is largely IGF-1-dependent, anti$P A P P A$ therapy might provide therapeutic effects beyond the inhibition of the IGF-1/IGF-1R signaling axis. A recent study indicated that IGFBP-4 could inhibit angiogenesis both induced by IGF-1 and FGF-2 [31], Therfore, given the fact that the inhibition of PAPPA can lead to the stabilization of IGFBP-4, targeting PAPPA might provide extra therapeutic benefits due to angiogenesis inhibition via IGFBP-4/FGF-2 interaction. In addition, there are evidences showing that PAPPA could promote angiogenesis in the chick chorioallantoic membrane assay via a possible nonproteolytic mechanism [32], further supporting the idea that PAPPA is not simply an alternative target for IGF-1 axis, but a novel target might provide extra therapeutic effects. Actually our preliminary data implied that nonproteolytic mechanisms might be involved in the function of PAPPA in MPM cells (data not shown). Now we are trying to validate these preliminary findings and further investigate the mechanistic role of PAPPA in the pathogenesis of MPM.

To conclude, our results supported the idea that PAPPA can function as a migration-promoting gene in MPM cells, and revealed that its migration-promoting role could be partially explained by its proteolytic activity. Given the fact that knockdown of PAPPA affected cell functions including cell migration in vitro and tumor development in vivo, we propose that it might be used a novel therapeutic target for the treatment of MPM.

\section{METHODS}

\section{Cell culture}

The human MPM cell lines, MSTO-211H, NCI-H2052, NCI-H2373, NCI-H2452 and NCI-H28, were purchased from the American Type Culture Collection (Manassas, VA). The human MPM cell lines, NCI-H290 and EHMES-1, were kindly provided by Dr. Adi F. Gazdar (University of Texas South-western Medical Center, Dallas, TX) and Dr. Hironobu Hamada (Ehime University, Toon, Japan), respectively. The human MPM cell lines, Y-MESO-14 and Y-MESO-5A, were established as described previously [33]. These MPM cell lines were maintained in RPMI1640 (Nissui Pharmaceutical, Tokyo, Japan) supplemented with $10 \%$ heat-inactivated fetal bovine serum (GIBCO, Grand Island, NY), penicillin (100 U/ml), and streptomycin $(50 \mu \mathrm{g} / \mathrm{ml})$. All cell lines were incubated at $37^{\circ} \mathrm{C}$ in a humidified atmosphere of $5 \%$ $\mathrm{CO}_{2}$. Cell lines were authenticated by DNA fingerprinting [34].

\section{Migration and invasion assay}

Cell migration was determined by using 24-well transwell chambers with polycarbonate membranes (8.0$\mu \mathrm{m}$ pore size; BD Biosciences, Bedford, MA). Human MPM cell suspensions in serum free medium were added to the upper chamber at $1 \times 10^{5}$ cells per well. RPMI- 
1640 culture medium with or without $10 \%$ FBS were added in the lower well, and the cells were allowed to migrate for $6 \mathrm{~h}$ at $37^{\circ} \mathrm{C}$ in $\mathrm{CO}_{2}$ incubator. Nonmigrant cells were removed from the upper face of the membrane with a cotton swab. The cells were then fixed and stained with Qiff-Quik Staining Kit (Sysmex, Kobe, Japan). The membranes were excised from the chamber and mounted. The migrant cells attached to the lower face of the membrane were counted. For in vitro selection of highly migratory cells, we used a method as descripted previously [35]. Briefly, instead of fixing and staining the migrated cells on the lower face of membrane as a routine process, the migrated cells were detached by trypsin treatment, then collected, cultured and propagated. The propagated cells were again subjected to transwell migration assay, and this process was repeated for 3-5 times. Cell Invasion was determined by using Matrigel-coated transwell chambers (BD Biosciences, Bedford, MA) according to protocols similar to that of migration assay except that allowing the cell to migrate for longer time $(18 \mathrm{~h})$.

\section{RNA isolation and quantitative RT-PCR}

Total cellular RNAs were isolated using RNeasy Mini Kit (Qiagen, Valencia, CA). After quantification and verification for purity (optical density 260/280 ratios between 1.8 and 2.0) with NanoDrop 1000 (Thermo Scientific, Yokohama, Japan), RNA was reversely transcribed with Takara PCR thermal cycler Dice (Takara Bio, Kyoto, Japan) using High Capacity RNA-to-cDNA Kit (Applied Biosystems, Foster City, CA) following the manufacturer's instruction. Subsequent quantitative RTPCR was carried out with CFX96 real-time PCR system (Bio-Rad, Hercules, CA) using Applied Biosystems' Taqman gene expression assays for PAPPA (Hs01032307 m1), IGFR1 (Hs00609566_m1), IGF1 (Hs01547656_m1), IGFBP1 (Hs00236877_m1), IGFBP2 (Hs01040719_m1), IGFBP3 (Hs00365742_g1), IGFBP4 (Hs01057900 m1), IGFBP5 (Hs00181213_m1). Samples were normalized to GAPDH (Hs99999905_m1) or ACTB (Hs99999903_ $\mathrm{m} 1)$ and quantification was determined by using the $\Delta \Delta \mathrm{CT}$ method.

\section{Microarray analysis}

Total RNA from MPM cells was isolated using an RNeasy Mini kit (QIAGEN, Valencia, CA). RNA quality was first checked for chemical purity using a NanoDrop spectrophotomer (NanoDrop Technologies, Wilmington, DE) and then assessed for RNA integrity using the Bioanalyzer 2100 (Agilent Technologies, Santa Clara, CA, USA). One hundred ng of total RNA was amplified and labeled using the Affymetrix Whole-Transcript (WT) Sense Target Labeling Protocol, and labeled RNA was hybridized to GeneChip Human Gene 1.0 ST Array
(Affymetrix, Santa Clara, CA). Data visualization and analysis was performed using GeneSpring GX (Version 12.1) software.

\section{ELISA}

The human MPM cells $\left(2 \times 10^{5}\right)$ were cultured in medium described above for $24 \mathrm{~h}$, washed with phosphate buffered saline (PBS), $3 \mathrm{ml}$ of fresh culture medium or serum free culture medium were added. After incubation for 24 or $48 \mathrm{~h}$, the culture media were harvested and centrifuged, and the supernatants were stored at $-70^{\circ} \mathrm{C}$ until analysis. Protein levels in supernatants were quantified by ELISA kit for human IGFBP-3, PAPPA (R\&D systems, Minneapolis, MN), IGFBP-4 (Abcam, Cambridge, MA) following the manufacturers' instructions.

\section{Immunoblotting analysis}

For the detection of IGFR1 expression in cell lines, the cells were lysed in M-PER (Pierce, Rockford, IL) containing phosphatase and proteinase inhibitor cocktails (Roche, Indianapolis, IN). The concentrations of protein were determined using Bio-Rad Protein Assay Kit (Bio-Rad, Hercules, CA). Blots were incubated with antibodies: IGFR1 (diluted 1:500, 111A9, Cell Signaling, Danvers, MA), $\beta$-actin (diluted1:5000, I-19, Santa Cruz Biotechnology, Santa Cruz, CA) overnight. For the detection of IGFBP-4 proteolysis, rhIGF-1, rhIGFBP-4, rhPAPPA and rhtPA (R\&D systems, Minneapolis, MN) were incubated at the indicated concentration in 50 $\mathrm{mM}$ Tris, $10 \mathrm{mM} \mathrm{CaCl}_{2}, 150 \mathrm{mM} \mathrm{NaCl}, \mathrm{pH} 7.5$ buffer for $6 \mathrm{~h}$ or overnight at $37^{\circ} \mathrm{C}$. Protein mixture was transferred to $\mathrm{iBlot}^{\mathrm{TM}}$ gel Tranfer Stacker polyvinylidene difluoride (PVDF) membrane (Invitrogen, Carlsbad, CA) according to manufacturer's instruction, the membranes were incubated at room temperature for $2 \mathrm{~h}$ with antiIGFBP-4 (K13RE) antibody (diluted 1:1000, Santa Cruz Biotechnology, Santa Cruz, CA). All analysis were revealed using the horseradish peroxidase(HRP)-labeled anti-rabbit or anti-mouse antibodies (GE Healthcare, Tokyo, Japan), and visualized using SuperSignal West Femto Maximum Sensitivity Substrate (Thermo Scientific, Yokohama, Japan) with a quantitative imaging system LAS4000Mini (Fujifilm, Tokyo, Japan).

\section{In vitro siRNA transfection}

Stealth RNAiTM siRNA for PAPPA (HSS107591, HSS181743; Invitrogen, Carlsbad, CA) transfection was conducted with Lipofectamine 2000 (Invitrogen, Carlsbad, CA) following the manufacture's protocol. Gene knock down efficacy was confirmed by qRT-PCR at the time 
points of 24, 48, and $72 \mathrm{~h}$. The resultant cells were used for biological assays at the indicated time point after siRNA transfection. Stealth RNAi ${ }^{\mathrm{TM}}$ siRNA Negative Control Med GC Duplex (Invitrogen, Carlsbad, CA) was used as negative control throughout the experiment.

\section{Lentivirus and infections}

MISSION shRNA pLKO.1 constructs (SigmaAldrich, St. Louis, MO) were used to make selfinactivating shRNA lentivirus for PAPPA [sequence 5'CCG GGG TGA CGG ATG GGA CAT ATT ACT CGA GTA ATA TGT CCC ATC CGT CAC CTT TTT TG-3' (clone NM_002581.3-3690s21c1)], and a non-target random scrambled sequence control (SHC002). For virus transduction, $2 \times 10^{6} \mathrm{NCI}-\mathrm{H} 290$ cells were incubated with lentivirus plus $5 \mu \mathrm{g} / \mathrm{ml}$ polybrene (CHEMICON International, Temecula, CA) for $24 \mathrm{~h}$. The successfully transduced clones were identified by puromycin (SigmaAldrich, St. Louis, MO) selection.

\section{MTT assay}

Cell proliferation was measured by the 3-(4,5-dimethylthiazol-2-yl)-2,5-diphenyltetrazolium bromide (MTT) dye reduction method [26]. Briefly, the MPM cells $\left(2 \times 10^{3} /\right.$ well $)$ were seeded in 96-well plates and cultured for $72 \mathrm{~h}$. After incubation, $50 \mu \mathrm{l}$ of stock MTT solution (2 mg/ml; Sigma, St. Louis, MO) was added to each well, and the cells were then further incubated for $2 \mathrm{~h}$. The media containing MTT solution were removed and the dark blue crystals were dissolved by adding $100 \mu \mathrm{l}$ of DMSO. Absorbance was measured with a Sunrise Microplate Reader (Tecan Group, Männedorf, Switzerland) at test and reference wavelengths of 550 and $620 \mathrm{~nm}$, respectively.

\section{Orthotopic xenograft mouse model}

Male severe combined immunodeficiency (SCID) mice aged 6-7 weeks (CLEA Japan, Osaka, Japan) were maintained under specific pathogen-free conditions throughout the study. Ethics approval for all animal experiments was obtained from Animal Care and Use Committee of The University of Tokushima. An orthotopic implantation model of human MPM was established as described previously [3-5, 26]. Briefly, $3 \times 10^{5}$ NCI-H290 cells in $100 \mu \mathrm{l}$ of PBS were injected into the right pleural cavity of mice. The mice were sacrificed 21-23 days after tumor inoculation. The pleural tumors were carefully dissected and weighed; the pleural effusion was harvested using a 1-ml syringe, and the volume of the pleural effusion was measured.

\section{Statistical analysis}

The statistical significances of the differences were analyzed by Student's $t$ test, one way ANOVA analysis, where applicable. The correlation efficient and the significance were calculated by Pearson's correlation analysis. The $P$-values less than 0.05 were considered significant in all experiments. Data analysis was carried out using GraphPad Prism version 5.01 (GraphPad Software, La Jolla, CA).

\section{ACKNOWLEDGEMENTS}

The authors thank Ms. Tomoko Oka for technical assistance. Y.N. receives research funding from Taiho Pharmaceutical.Co., Ltd. This work was supported in part by research grant from Taiho Pharmaceutical. Co., Ltd, KAKENHI (24390210, 22700882), a Grant-in-Aid for Scientific Research (Y.N.), and a Challenging Exploratory Research (S.K.) from the Ministry of Education, Culture, Sports, Science and Technology (MEXT) of Japan.

\section{REFERENCES}

1. Robinson BW, Lake RA. Advances in malignant mesothelioma. N Engl J Med. 2005; 353: 1591-1603.

2. Ray M, Kindler HL. Malignant pleural mesothelioma: an update on biomarkers and treatment. Chest. 2009; 136: 888896.

3. Li Q, Yano S, Ogino H, Wang W, Uehara H, Nishioka Y, Sone S. The therapeutic efficacy of anti-vascular endothelial growth factor antibody, bevacizumab, and pemetrexed against orthotopically implanted human pleural mesothelioma cells in severe combined immunodeficient mice. Clin Cancer Res. 2007; 13: 5918-5925.

4. Ikuta K, Yano S, Van TT, Hanibuchi M, Goto H, Li Q, Wang W, Yamada T, Ogino H, Kakiuchi S, Uehara H, Sekido Y, Uenaka T, Nishioka Y, Sone S. E7080, a multityrosine kinase inhibitor, suppresses the progression of malignant pleural mesothelioma with different proangiogenic cytokine production profiles. Clin Cancer Res. 2009; 15: 7229-7937.

5. Van TT, Hanibuchi M, Goto H, Kuramoto T, Yukishige S, Kakiuchi S, Sato S, Sakaguchi S, Dat LT, Nishioka Y, Akiyama S, Sone S. SU6668, a multiple tyrosine kinase inhibitor, inhibits progression of human malignant pleural mesothelioma in an orthotopic model. Respirology. 2012; 17: 984-990.

6. Jakobsen JN, Sørensen JB. Review on clinical trials of targeted treatments in malignant mesothelioma. Cancer Chemother Pharmacol. 2011; 68: 1-15.

7. West SD, Lee YC. Management of malignant pleural mesothelioma. Clin Chest Med. 2006; 27: 335-354.

8. Wells A, Grahovac J, Wheeler S, Ma B, Lauffenburger D. 
Targeting tumor cell motility as a strategy against invasion and metastasis. Trends Pharmacol Sci. 2013; 34: 283-289.

9. Lin TM, Galbert SP, Kiefer D, Spellacy WN, Gall S. Characterization of four human pregnancy-associated plasma proteins. Am J Obstet Gynecol. 1974; 118: 223-236.

10. Lawrence JB, Oxvig C, Overgaard MT, Sottrup-Jensen L, Gleich GJ, Hays LG, Yates JR III, Conover CA. The insulin-like growth factor (IGF)-dependent IGF binding protein-4 protease secreted by human fibroblasts is pregnancy associated plasma protein-A. Proc Natl Acad Sci USA. 1999; 96: 3149-3153.

11. Boldt HB, Conover CA. Pregnancy-associated plasma protein-A (PAPP-A): A local regulator of IGF bioavailability through cleavage of IGFBPs. Growth Horm IGF Res. 2007; 17: 10-18.

12. Gyrup C, Oxvig C. Quantitative analysis of insulin-like growth factor-modulated proteolysis of insulin-like growth factor binding protein- 4 and -5 by pregnancy associated plasma protein-A. Biochemistry. 2007; 46: 1972-1980.

13. Pollak M. Insulin and insulin-like growth factor signalling in neoplasia. Nature Rev Cancer. 2008; 8: 915-928.

14. Hoang CD, D’Cunha J, Kratzke MG, Casmey CE, Frizelle SP, Maddaus MA, Kratzke RA. Gene expression profiling identifies matriptase overexpression in malignant mesothelioma. Chest. 2004; 125: 1843-1852.

15. Hoang CD, Zhang X, Scott PD, Guillaume TJ, Maddaus MA, Yee D, Kratzke RA. Selective activation of insulin receptor substrate-1 and -2 in pleural mesothelioma cells: association with distinct malignant phenotypes. Cancer Res. 2004; 64: 7479-7485.

16. Jacobson BA, De A, Kratzeke MG, Patel MR, Jay-Dixon J, Whitson BA, Sadiq AA, Bitterman PB, Polunovsky VA, Kratzke RA. Activated 4E-BP1 represses tumourigenesis and IGF-I-mediated activation of the eIF4F complex in mesothelioma. Br J Cancer. 2009; 101: 424-431.

17. King ER, Wong KK. Insulin-like growth factor: current concepts and new developments in cancer therapy. Recent Pat Anticancer Drug Discov. 2012; 7: 14-30.

18. Conover CA, Bale LK. Loss of pregnancy-associated plasma protein A extends lifespan in mice. Aging Cell. 2007; 6: 727-729.

19. Conover CA, Bale LK, Mader JR, Mason MA, Keenan KP, Marler RJ. Longevity and age-related pathology of mice deficient in pregnancy-associated plasma protein-A. J Gerontol A Biol Sci Med Sci. 2010; 65: 590-599.

20. Ryan AJ, Napoletano S, Fitzpatrick PA, Currid CA, O'Sullivan NC, Harmey JH. Expression of a proteaseresistant insulin-like growth factor-binding protein-4 inhibits tumour growth in a murine model of breast cancer. Br J Cancer. 2009; 101: 278-286.

21. Boldt HB, Conover CA. Overexpression of pregnancyassociated plasma protein-A in ovarian cancer cells promotes tumor growth in vivo. Endocrinology. 2011; 152: $1470-1478$.
22. Pan H, Hanada S, Zhao J, Mao L, Ma MZ. Protein secretion is required for pregnancy-associated plasma protein-A to promote lung cancer growth in vivo. PLoS One. 2012; 7: e48799.

23. Kalra N, Zhang J, Yu Y, Ho M, Merino M, Cao L, Hassan R. Efficacy of anti-insulin-like growth factor I receptor monoclonal antibody cixutumumab in mesothelioma is highly correlated with insulin growth factor-I receptor sites/ cell. Int J Cancer. 2012; 131: 2143-2152.

24. Bang P. Serum proteolysis of IGFBP-3. Prog Growth Factor Res. 1995; 6: 285-292.

25. Liu Z, Klominek J. Chemotaxis and chemokinesis of malignant mesothelioma cells to multiple growth factors. Anticancer Res. 2004; 24: 1625-1630.

26. Van TT, Hanibuchi M, Kakiuchi S, Sato S, Kuramoto T, Goto H, Mitsuhashi A, Nishioka Y, Akiyama S, Sone S. The therapeutic efficacy of S-1 against orthotopically implanted human pleural mesothelioma cells in severe combined immunodeficient mice. Cancer Chemother. Pharmacol. 2011; 68: 497-504.

27. Lee TC, Zhang Y, Aston C, Hintz R, Jagirdar J, Perle MA, Burt M, Rom WN. Normal human mesothelial cells and mesothelioma cell lines express insulin-like growth factor I and associated molecules. Cancer Res. 1993; 53: 28582864.

28. Gordon GJ, Rockwell GN, Jensen RV, Rheinwald JG, Glickman JN, Aronson JP, Pottorf BJ, Nitz MD, Richards WG, Sugarbaker DJ, Bueno R. Identification of novel candidate oncogenes and tumor suppressors in malignant pleural mesothelioma using large-scale transcriptional profiling. Am J Pathol. 2005; 166: 1827-1840.

29. Friedrich N, Alte D, Völzke H, Spilcke-Liss E, Lüdemann J, Lerch MM, Kohlmann T, Nauck M, Wallaschofski H. Reference ranges of serum IGF-1 and IGFBP-3 levels in a general adult population: Results of the Study of Health in Pomerania (SHIP). Growth Horm IGF Res. 2008; 18: 228237.

30. Su AI, Wiltshire T, Batalov S, Lapp H, Ching KA, Block D, Zhang J, Soden R, Hayakawa M, Kreiman G, Cooke MP, Walker JR, Hogenesch JB. A gene atlas of the mouse and human protein-encoding transcriptomes. Proc Natl Acad Sci USA. 2004; 101: 6062-6067.

31. Contois LW, Nugent DP, Caron JM, Cretu A, Tweedie E, Akalu A, Liebes L, Friesel R, Rosen C, Vary C, Brooks PC. Insulin-like growth factor binding protein-4 (IGFBP-4) differentially inhibits growth factor-induced angiogenesis. J Biol Chem. 2012; 287: 1779-1789.

32. Jadlowiec J, Dongell D, Smith J, Conover C, Campbell P. Pregnancy-associated plasma protein-A is involved in matrix mineralization of human adult mesenchymal stem cells and angiogenesis in the chick chorioallontoic membrane. Endocrinology. 2005; 146: 3765-3772.

33. Kawaguchi K, Murakami H, Taniguchi T, Fujii M, Kawata S, Fukui T, Kondo Y, Osada H, Usami N, Yokoi K, Ueda 
Y, Yatabe Y, Ito M, Horio Y, Hida T, Sekido Y. Combined inhibition of MET and EGFR suppresses proliferation of malignant mesothelioma cells. Carcinogenesis. 2009; 30: 1097-1105.

34. Abe S, Morita Y, Kaneko MK, Hanibuchi M, Tsujimoto Y, Goto H, Kakiuchi S, Aono Y, Huang J, Sato S, Kishuku M, Taniguchi Y, Azuma M, Kawazoe K, Sekido Y, Yano S, et al. A Novel targeting therapy of malignant mesothelioma using anti-podoplanin antibody. J Immmunol. 2013; 190: 6239-6249.

35. Gunawardane RN, Sgroi DC, Wrobe CN, Koh E, Daley GQ, Brugge JS. Novel role for PDEF in epithelial cell migration and invasion. Cancer Res. 2005; 65: 11572-11580. 